Review Article

\title{
Therapeutic Effects of Ten Commonly Used Chinese Herbs and Their Bioactive Compounds on Cancers
}

\author{
Wei Liu $\mathbb{D}^{1,2}$ Binbin Yang, ${ }^{1,2}$ Lu Yang, ${ }^{1,2}$ Jasmine Kaur, ${ }^{2}$ Calvin Jessop, ${ }^{2}$ Rushdi Fadhil, ${ }^{2}$ \\ David Good $\mathbb{D}$, ${ }^{2,3}$ Guoying Ni $\left(\mathbb{D},{ }^{2,4,5,6}\right.$ Xiaosong Liu $\left(\mathbb{D},{ }^{4,5,6}\right.$ Tamim Mosaiab, ${ }^{2}$ Zhengjun Yi $\mathbb{D}^{1},{ }^{1}$ \\ and Ming Q. Wei $\mathbb{D}^{2}$ \\ ${ }^{1}$ Department of Laboratory Medicine, Key Laboratory of Clinical Laboratory Diagnostics in Universities of Shandong, \\ Weifang Medical University, 261053 Weifang, Shandong, China \\ ${ }^{2}$ School of Medical Science \& Menzies Health Institute Queensland, Griffith University, Brisbane, Australia \\ ${ }^{3}$ School of Allied Health, Australian Catholic University, Brisbane, Australia \\ ${ }^{4}$ Cancer Research Institute, Guangdong Pharmaceutical University, Guangzhou, China \\ ${ }^{5}$ Inflammation and Healing Research Cluster, Sunshine Coast University, Sunshine Coast, Australia \\ ${ }^{6}$ Molecular Diagnosis and Target Therapy Laboratory, Guangzhou, China
}

Correspondence should be addressed to Zhengjun Yi; fuyizhengjun@163.com and Ming Q. Wei; m.wei@griffith.edu.au

Received 20 May 2019; Revised 28 July 2019; Accepted 27 August 2019; Published 15 September 2019

Academic Editor: Svein Haavik

Copyright ( $\odot 2019$ Wei Liu et al. This is an open access article distributed under the Creative Commons Attribution License, which permits unrestricted use, distribution, and reproduction in any medium, provided the original work is properly cited.

Effective cancer therapy is one of the biggest global challenges. Conventional cancer therapies have been at the forefront of combating cancers, but more evidence showed considerable side effects, limiting their use. There are various new therapies in development, but combined approaches for treating cancer are much expected. Natural herbs had been traditionally in use for cancer therapy in most parts of the world. In this review, we have examined ten commonly used Chinese herbs that have, for centuries, shown effectiveness in treating cancers. They demonstrated the abilities to promote the apoptosis of cancer cells, inhibit their metastasis, activate the patient's anticancer immunity, and synergistically increase the efficacy of conventional chemotherapy and radiation therapy when used in combination. Clinical experiences had proved that these herbs and their bioactive compounds were effective against a plethora of cancers through a variety of mechanisms, effectively improving patients' quality of life without significant side effects. These advantages indicate that there are huge potentials in the development of Chinese herbs into cancer medicine as part of a promising, holistic cancer treatment modality.

\section{Introduction}

Cancer is the leading cause of death worldwide, with an estimated 9.6 million people dying of cancer in 2018 [1]. It is groups of uncontrolled, cell proliferative, and chronic diseases, which pose an immediate threat to the life of patients with huge healthcare expenses [2]. Surgery, radiotherapy, and chemotherapy are now the most mainstreams of cancer therapy coupled with the emerging targeted and cancer immunotherapies [3]. To a certain extent, the growth and spread of cancer were controlled and the survival time of patients was prolonged by these methods, but the overall efficacy and many of the associated side effects were intolerable. In some cases, these treatments are effective, but the therapeutic effects did not last long, or cancer cells gained resistance to chemical drugs, limiting their further use. Therefore, it is necessary to develop a more ideal drug therapeutics or adjuvant treatment strategy for cancer.

Chinese herbs have demonstrated efficacy in the treatment and prevention of cancer through thousands of years of practice [4]. As a natural medicine, it has the advantages of low adverse reaction and low toxicity. There are over 2000 herbs registered in the Chinese Herbal register, which had been used of the treatment of a range of illnesses; about 400 have varying degrees of therapeutic effects on cancers, such as those of the lung, liver, brain, stomach, prostate, and breast [5]. The mechanisms may relate to the inhibition of cancer growth, reduction of metastasis and invasion, promotion of 
cancer cell apoptosis, and enhancement of immunity. Chinese herbal medicine is increasingly used as an alternative to mainstream cancer treatments [6]. Combination therapy with the use of herbs had produced more effective and lasting therapeutic effects. Furthermore, herbs can sensitize the body to radiotherapy and chemotherapy, increase the protection of normal tissues, and prevent tumor metastasis and recurrence. Chinese herbal medicine can not only inhibit local lesions but also regulate the whole body to eliminate cancers, without damage to normal cells [6]. In addition, Chinese herbal medicine can reduce the side effects of radiotherapy and chemotherapy, alleviate insomnia, depression, and fatigue, and significantly improve the quality of life. Therefore, herbs have unique advantages in improving the quality of life and extending the survival period of patients $[6,7]$.

The purpose of this article is to review the anticancer effects of ten commonly used Chinese herbs and their bioactive compounds for their abilities in enhancing immunity and killing cancer cells, thus providing rationales for the development of novel, strategic treatments for cancers.

\section{Ten Commonly Used Herbs}

There are numerous herbs and their many remedies/recipes, which have been used for cancer therapy through the past 2000 years in China and South and East Asia [8]. The mechanisms and effects are vastly different. In this review, we searched the top ten Chinese herbs with the highest number of results related to cancer on China National Knowledge Infrastructure (CNKI) and were presented here. Each herb has good anticancer properties and is widely used as cancer therapeutics.

2.1. Oldenlandia diffusa (OD, Bai Hua She She Cao in Chinese). OD as an annual herb has a wide range of pharmacological effects, including antioxidant, anticancer and immune regulation (Figure 1). It is one of the most wellknown Chinese herbs for its anticancer effect. Chemical analysis has revealed that bioactive compounds including vanillic acid, 2-hydroxy-3-methyl-anthraquinone, 2-hydroxy-7-methyl-3methoxyanthraquinone, and 1-formaldehyde-4-hydroxyl anthraquinone are the main anticancer components of OD [7]. Vanoxalic acid and 2-hydroxy-3methyl-anthraquinone are good tyrosinase inhibitors which have been used clinically to fight cancer. For clinical use, the ethanol-extracted OD had better efficacy.

$\mathrm{OD}$ and its active components can effectively induce apoptosis of prostate cancer, colorectal cancer, cervical cancer, gastric cancer, and other tumor cells [9]. It can reduce the proliferation and metastasis of liver cancer cells by inhibiting the expression of chemokine receptors such as CXCR1, CXCR2, and CXCR4 and induce apoptosis through caspase 3 pathway [10]. Chung et al. [11] found that OD inhibits the expression of MMP-9 to inhibit the invasion of breast cancer cells and regulates the expression level of apoptosis-related proteins to induce apoptosis of cancer cells. In addition, the water extract of OD leads to the apoptosis of lung cancer cells by inhibiting the MAPK pathway [5]. The

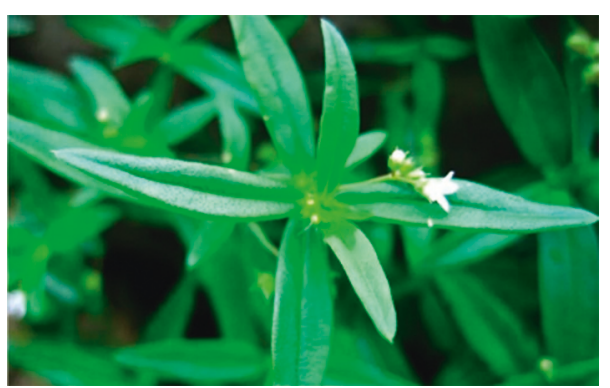

FIgUre 1: Photo of an Oldenlandia diffusa plant, with leaves and flowers.

expressions of apoptosis-related proteins Fas, caspase 3, and caspase 7 were significantly increased, and the expression of Fas L protein was inhibited in the kidney cancer model mice with the treatment of OD [12]. It can also induce apoptosis and inhibit the proliferation of bladder cancer T24 cells through the JAK2/STAT3 pathway [13]. In vivo experiments showed that total flavonoids of OD could significantly inhibit the tumor growth of cervical cancer in mice. The mechanism may be related to the increase of serum TNF- $\alpha$, IFN- $\gamma$, and IL2 levels [14]. Furthermore, the clinical application has proved that the herb can also achieve the effect of cancer control by enhancing body immunity [15].

As an anticancer drug, OD has a long history of clinical use with a good inhibitory effect on a variety of cancers, but it is often used in combination with other drugs to enhance its anticancer effects, most commonly in combination with $\mathrm{Scu}$ tellaria barbata. It can be the best herb for antitumor therapy.

2.2. Curcuma longa (CL, Jiang Huang in Chinese). CL, known as turmeric, is a kind of perennial herb (Figure 2). Its main active ingredient is curcumin which has been used as a spice and pigment. Curcumin is a kind of diketone compound that has few side effects in humans. As a therapeutic agent, it is also used for the treatment of cancers and chronic diseases such as cardiovascular disease and diabetes. Turmeric is often taken as a single decoction.

Curcumin can inhibit the proliferation and migration of cervical cancer cells and cause the apoptosis of metastatic cells without affecting the survival of normal cervical epithelial cells [16]. Curcumin can also reverse TGF-induced epithelial-mesenchymal transformation (EMT) in hepatocellular carcinoma by downregulating Snail expression [17]. It has been reported that curcumin can inhibit the proliferation and migration of glioma cells and promote their apoptosis and its mechanism is closely related to the inhibition of the expression of oncogenic protein NEDD4 [18]. Similarly, curcumin has been found to affect the proliferation, invasion, and apoptosis of pancreatic cancer cells by inhibiting NEDD4 [19]. Curcumin may inhibit the expression of Cyclin B1 and downregulate the apoptosis-related protein $\mathrm{Bcl}$-xl resulting in inhibition of the proliferation of thyroid cancer and promoting apoptosis [20]. Besides, curcumin significantly reduces the activity of oral squamous cell carcinoma cells by inhibiting the PI3K/AKT/mTOR signaling pathway 


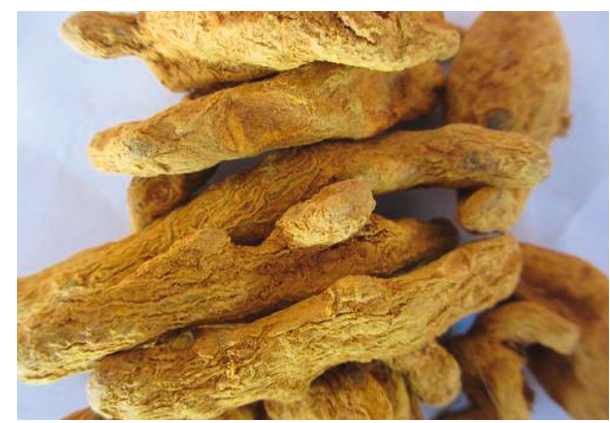

Figure 2: Photo of dry root Curcuma longa.

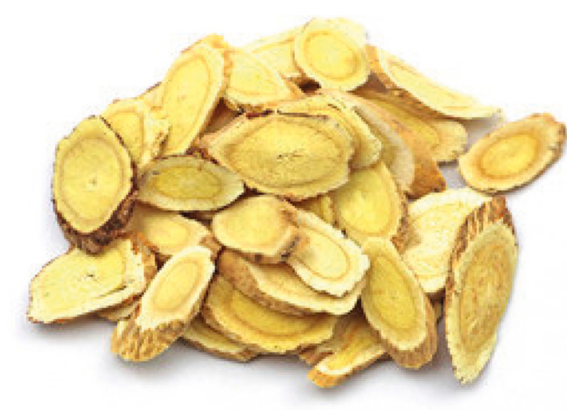

FIgUre 3: Photo of dry root Astragalus membranaceus slices.
[21]. In vivo experiment showed that gavage of curcumin to cervical cancer mice can effectively reduce the level of tumor marker molecule and nitric oxide, indicating the inhibition of cancer growth [22].

Turmeric is often used as a single drug to suppress the activity of different types of cancer, especially cancer of the digestive system from multiple perspectives. In recent years, curcumin has been proved to be an excellent natural compound against cancer, especially in the digestive system. So far, there are no reports that curcumin is toxic to animals or humans. These unique advantages of turmeric make it an excellent choice for the treatment and prevention of cancer.

\subsection{Astragalus membranaceus (AM, Huang Qi in Chinese).} $\mathrm{AM}$ is a very common Chinese medicine in China, belonging to the leguminous plant (Figure 3). Its main medicinal ingredients are astragalus polysaccharide and astragaloside, which have good immune and antiviral functions [23]. Astragalus polysaccharide is the most active ingredient in astragalus, and the higher concentration of ethanol extract has stronger activity. Astragalus injection is the most commonly used method of AM. It is also one of the main components of Buzhong Yiqi decoction, which is a traditional Chinese medicine prescription with good effect on cancers.

Studies have found that astragaloside can downregulate the expression of immune checkpoint proteins PD-1 and PD-L1 and inhibit the expression levels of migration-related proteins MMP-2 and MMP-9, which may be related to the inhibition of invasion and migration of cervical cancer cells $[24,25]$. AM can also curb the expression of MMP-2 and MMP-9 proteins and inhibit the phosphorylation of ERK to control the proliferation and metastasis of ovarian cancer. AM can regulate the apoptosis of cancer cells by upregulating the apoptosis-related proteins caspase 3 and caspase 9 and increasing the proportion of Bax/Bcl-2 [26]. Zhou et al. [27] found that AM inhibits the proliferation of breast cancer cells through the PI3K/AKT/mTOR pathway and induce apoptosis of non-small cell lung cancer [28] and leukemia [29]. AM can also induce the polarization of macrophages into M1-type and activate the anticancer ability of macrophages [30]. Moreover, AM combined with other anticancer drugs such as cisplatin can significantly increase the inhibitory effect on cancer cells [31].

In recent years, AM has attracted much attention because of its good anticancer activity. AM has an obvious inhibitory effect on cervical cancer, breast cancer, lung cancer, and leukemia. AM can not only be taken as a single medicine but also used to synergistically enhance the efficacy of cisplatin. Therefore, it can be used as one of the research directions of anticancer herbs in the future.

2.4. Panax ginseng ( $P G$, Ren Shen in Chinese). PG is the dry rhizome of the Araliaceous plant ginseng, which has attracted much attention due to its multiple pharmacological functions such as cardiovascular protection, antitumor, and antiaging [32] (Figure 4). Ginsenoside is the main active ingredient of ginseng, and some polysaccharides and amino acids also have some pharmacological functions. Ginsenosides are triterpenoids which have anticancer activities mainly include Rg1, Rg2, Rg3, Rh1, and Rh2. Ginseng is usually taken alone and is also the main ingredient of Lizhong decoction.

It is reported that $\mathrm{Rh} 2$ ginsenosides can promote endometrial cancer cells apoptosis, and its mechanism may be related to increased apoptosis markers cleaved poly ADPribose polymerase (PARP) and caspase 3 protein levels [33]. Ginsenoside Rg5 can regulate the miR125b/STARD13/ NEU1 signaling pathway, which may be one of the mechanisms of ginseng in inhibiting invasion and migration of gastric cancer cells [34]. Meanwhile, ginsenoside Rg5 can also reduce $\mathrm{Bcl}-2$ protein and increase the expression of Bax protein in gastric cancer cells, thereby reducing the activity of cancer cells [35]. When studying lung cancer cell A549, ginsenoside $\operatorname{Rg} 3$ induced apoptosis through upregulation of ROS formation and apoptosis protein caspase $3 / 9$ and BAX [36]. Additionally, ginsenoside CK also has a good effect in inhibiting breast cancer invasion and metastasis and its mechanism is related to the downregulation of MMP-2 and MMP-9 expression [37]. Recently, it has been found that PG and astragalus water extract can regulate the polarization of macrophages toward M1-type and synergistically enhance the anticancer effect of cis-diamine dichloroplatin (DDP) [38]. In vivo experiments found that ginsenoside Rh 2 can regulate the immune function of mice and significantly improve the killing effect of CD8+ T cells and NK cells on cancer cells [39].

As a star herb, PG has been widely used in Asia especially East Asia for thousands of years. In addition to its excellent anticancer properties, ginseng's immunomodulatory properties make it a good choice for anticancer herbs. 


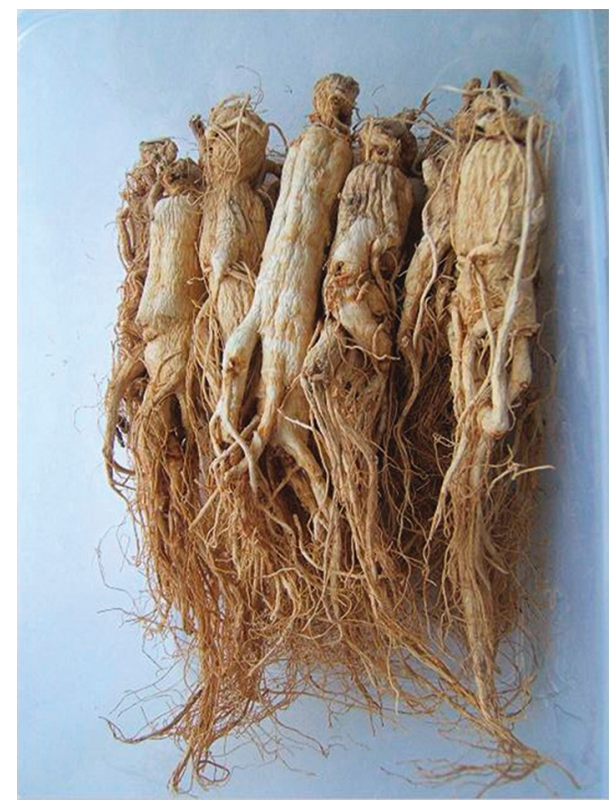

Figure 4: Photo of dry root Panax ginseng.

2.5. Ganoderma lucidum (GL, Ling Zhi in Chinese). GL is a kind of fungus with both food and medicine (Figure 5). Besides its good health care function, GL can also manage diabetes and improve cardiovascular and anti-aging pharmacological effects. The active components of GL include polysaccharides, triterpenoids, proteins, amino acids, sterols, and alkaloids [40]. The polysaccharide is composed of three single sugar chains, with a spiral three-dimensional configuration of dextran; the three-dimensional configuration is similar to deoxyribonucleic acid DNA and RNA and is the most effective component of GL. GL is generally taken as a single drug, as a tonic drink, or food to take.

It has been reported that Ganoderma triterpenoids can inhibit the proliferation of lung cancer cells and the molecular mechanism is related to the regulation of cell cycle and the increase of $\mathrm{Bax} / \mathrm{Bcl}$ ratio [41]. Triterpenoids can also downregulate MMPs to inhibit metastasis of prostate cancer cells and reduce cancer activity [42]. In breast cancer, GL extract reduces the number of breast cancer stem cells (BCSCs) by downregulating the STAT3 pathway to inhibit the invasion ability of cancer cells [43]. Furthermore, it has been reported that GL extracted with ethanol can enhance the protective autophagy of cells and inhibit the expression of EGFR and the $\mathrm{PI} 3 \mathrm{~K} / \mathrm{AKT} / \mathrm{mTOR}$ signaling pathway in chronic myelogenous leukemia cells [44]. Besides, GL polysaccharides can assist the anticancer effect of the chemotherapy drug paclitaxel (PTX) and inhibit the cancer metabolic process in the cancer microenvironment [45]. In addition to the direct killing of cancers, GL can also be used in immunotherapy against cancers. Studies have shown that GL polysaccharidegold nanocomposite can activate dendritic cells and promote the proliferation of T cells and has a strong inhibitory effect on the proliferation and metastasis of lung cancer cells [46].

As a traditional Chinese medicine with both food and medicine, GL can be consumed by cancer patients in their daily diet. The effective ingredients in GL are very effective in

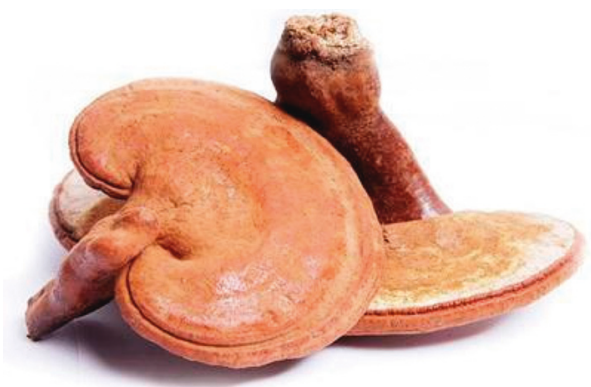

FIgUre 5: Photo of Ganoderma lucidum.

the treatment of lung cancer, prostate cancer, breast cancer, and so on. GL can be used as an herbal treatment for cancer that is more easily accepted by patients.

2.6. Angelica sinensis (AS, Dang Gui in Chinese). AS belongs to perennial herbaceous plant, has extensive pharmacological action, has good cardiovascular, analgesic, and antiinflammatory effects (Figure 6). It has many components, among which volatile oil, organic acid, and polysaccharides are the main [47]. The volatile oil is mainly composed of Z-ligustilide, which has anticancer and immunity-enhancement effect. AS is commonly used as a single food. Danggui buxue decoction is often used for the treatment of tumor-related anemia, which consists of AS and AM.

In studies on bladder cancer, it is found that N-butylidenephthalide extracted from Angelica inhibits the activity of cancer cells from multiple aspects, including upregulating caspase 3/9 to induce apoptosis of cancer cells, upregulating E-cadherin, and downregulating $\mathrm{N}$-cadherin to inhibit cell migration. In combination with cisplatin, it can increase the sensitivity of cancer cells [48]. Angelica acetone extract significantly reduced the expression of hypoxia-inducible factor 1-alpha (HIF-1- $\alpha$ ) and vascular endothelial growth factor (VEGF) in bladder cancer cells, effectively reducing the formation of cancer microenvironment. Meanwhile, the $\mathrm{PI} 3 \mathrm{~K} / \mathrm{AKT} / \mathrm{mTOR}$ signaling pathway of cancer cells is also inhibited [49]. Angelica polysaccharides induced breast cancer cells to express the cAMP-responsive elementbinding protein (CREB), upregulated caspase 3/9, and cleaved PARP and led to apoptosis of cancer cells [50]. Studies have shown that high concentration of AS can inhibit the metastasis of lung cancer cells and the possible mechanism is not only to reduce the expression of MMP2, MMP-9, TGF-1, and metalloproteinase tissue inhibitor TIMP-1 but to increase the expression of TIMP-2 [51]. In addition, Angelica polysaccharide downregulates cyclins and Bcl-2, upregulates Bax, cleaves caspase 3 and E-cadherin, inhibits the TGF-signaling pathway and the growth activity of glioma cells in vivo and in vitro [52].

AS is often used in traditional Chinese medicine for cancer treatment and is an excellent choice for gynecological cancers such as breast cancer.

2.7. Panax notoginseng ( $P N$, San Qi in Chinese). PN is the dry root tuber of Panax notoginseng, which has strong 


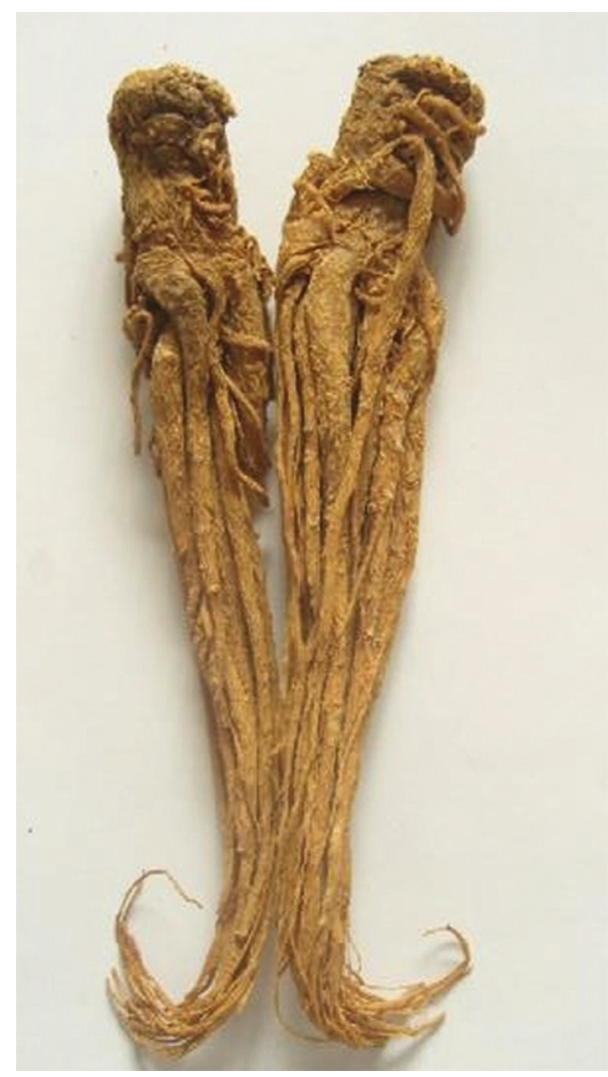

Figure 6: Photo of dry root Angelica sinensis.

pharmacological functions of enhancing immunity, antioxidation, antitumor, and antiaging (Figure 7). The main active components are Panax notoginseng saponins (PNS), in which ginsenoside Rb1, Rg1, Re, Rd, and PNS R1 play the main functions $[53,54]$. In recent years, more and more studies have confirmed the efficacy of PN in the treatment of cancer, including the improvement of immunity and the destruction of the function of cancer cells. Powder made from $\mathrm{PN}$ is commonly used in traditional Chinese medicine.

It has been reported that Panax notoginseng ethanol extract (PNEE) can inhibit the invasion and metastasis of colorectal cancer cell line (HCT-116) by reducing MMP-9 and increasing the expression of E-cadherin, thus maintaining the integrity of the intercellular matrix. Also, PNEE reduces the protein levels of integrin-1 in HCT-116 and E-selectin and intercellular adhesion molecule-1 (ICAM-1) in endothelial cells EA.hy926, which reduces the adhesion ability of cancer cells [55]. PNS significantly inhibits the metastasis of breast cancer cells, and its function is to upregulate the expression of E-cadherin and cancer suppressor genes Brms1, Mtss1, and Timp2 and downregulate the expression of MMP3, MMP-9, and vimentin [56]. The growth of Lewis lung cancer cell line (LLC) treated with PNS was significantly reduced, and the expression level of Met/ miR-222 axis was significantly reduced. And the expressions of cancer suppressor p27 and PTEN, which are the target genes of miR-222, were increased both in vivo and in vitro [57]. Furthermore, PNS can increase the cytotoxicity of cisplatin by enhancing the gap junction intercellular

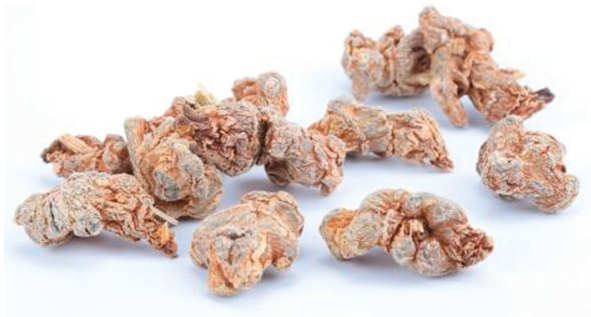

Figure 7: Photo of dry root Panax notoginseng.

communication [58]. PN could regulate the polarization of macrophage $\mathrm{M} 1$, which can induce apoptosis of lung cancer cells by upregulating the apoptotic protein caspase $3 / 9$, to reduce the volume of solid cancers [59].

Panax notoginseng can inhibit the activity of cancer cells through various mechanisms, enhance the immune cell function of cancer patients, and increase the efficacy of chemotherapy drugs. It has good effects on colorectal cancer, breast cancer, and lung cancer, and so on. It can help patients fight cancer in many aspects.

\subsection{Scutellaria barbata D. Don (SB, Ban Zhi Lian in Chinese).} SB is the whole herb of Scutellaria barbata, a perennial herb in the family Lamiaceae, which shows good efficacy in antitumor, antivirus, and antioxidation properties (Figure 8). The main active components of the herb are flavonoids, diterpenoids, and polysaccharides [60]. Scutellarin is the main ingredient in $10 \%$ of flavonoids. In traditional Chinese medicine theory, SB and OD are both heat-clearing and detoxifying drugs, so they are generally used in combination.

Studies on ovarian cancer cells showed that SB extracts downregulated Bcl-2 protein and increased caspase 3/9 protein to induce apoptosis. The migration ability of cancer cells was also inhibited, which may be closely related to the decreased expression of MMP-2/9 [61]. It inhibits the expression of HIF-1 and VEGF to inhibit the formation of cancer microenvironment and has been shown in vivo to inhibit the development of lung cancer and reduce capillary density [62]. Besides, polysaccharides of SB can increase the levels of Bax, Bak protein, and E-cadherin, decrease the levels of $\mathrm{Bcl}-2$ protein, $\mathrm{N}$-cadherin, and vimentin, inhibit the proliferation and EMT of colon cancer cells, and promote their apoptosis [63]. Studies have shown that the combined application of SB and OD can promote the apoptosis of bladder cancer cells by downregulating the expression of miR-155 and its regulated Akt signaling pathway. Meanwhile, the expression of McL-1 and Bcl-2 was also inhibited, and the expression of caspase 3 was upregulated [64]. In vivo experiments on mice showed that the ethanol extract of Scutellaria barbata (EESB) significantly reduced the expression of the Ki-67 protein, a cell proliferation marker of colorectal cancer. Also, the $\mathrm{Wnt} / \beta$-catenin signaling pathway, proto-oncogene $\mathrm{c}$-myc, and antiapoptotic protein survivin are all inhibited [65]. Moreover, EESB inhibits il-6mediated STAT3 activation in colorectal cancer cells and downregulates the expression of cyclin D1 and CDK4 [66]. 


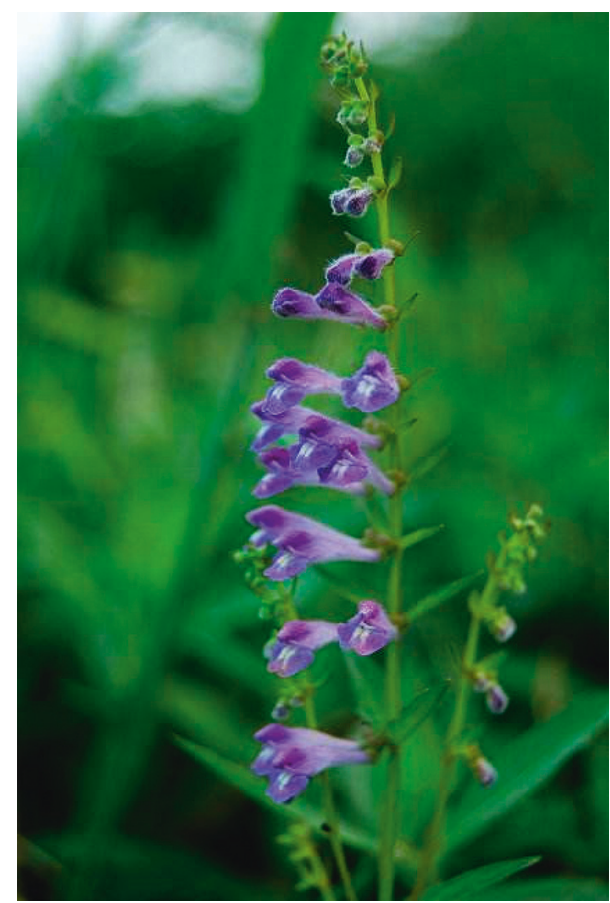

Figure 8: Photo of a Scutellaria barbata D. Don plant, with leaves and flowers.

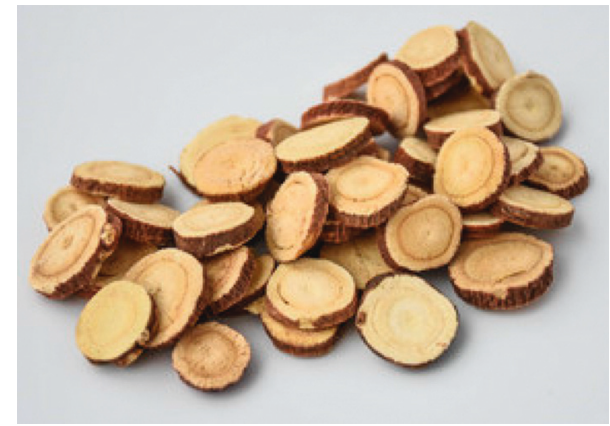

Figure 9: Photo of dry root licorice slices.

An experiment on C26 tumor-bearing mice showed that SB polysaccharide can inhibit tumor growth by activating caspase 3/9 [67].

The combination of Scutellaria barbata and Oldenlandia diffusa can enhance the anticancer effect of each other, which is the key point of anticancer herbs. It mainly treats digestive tract cancers, gynecological cancers, lung cancer, bladder cancer, and other cancers.

2.9. Licorice (Gan Cao in Chinese). Licorice is the dry root and rhizome of the leguminous plant Glycyrrhiza uralensis (Figure 9). The main active ingredient of licorice is glycyrrhizic acid (or glycyrrhizin), which is a saponin structurally. Licorice has a clinical application history of more than 2,000 years and has a variety of pharmacological effects such as antibacterial, antiviral, anti-inflammatory and antitumor [68]. Licorice tablet is the most commonly used form.
It is reported that mitochondrial ROS production in breast cancer cells treated with glycyrrhizin was significantly increased. Meanwhile, the expressions of the apoptosis-inducing factor (AIF) and the autophagy protein LC-3 were upregulated, indicating that glycyrrhizic acid simultaneously regulates the apoptosis and autophagy process of cancer cells and mediates their death [69]. Glycyrrhizin can induce apoptosis of oral cancer cells by increasing the ratio of Bax/ Bcl-2 and upregulating the cleavage of caspase 3/9 and PARP [70]. In addition, $18 \beta$-glycyrrhizic acid can inhibit the metastasis and invasion of gastric cancer cells, which may be related to the decreased expression of MMP-2/9 and vimentin, and upregulate the expression of E-cadherin. At the same time, this study found that 18 glycyrrhetinic acids could inhibit EMT of cancer cells by inhibiting ROS/pkc-/ ERK signaling pathway [71]. Moreover, the protein extract of licorice also inhibited the activity of gastrointestinal cancer cells to some extent but had no significant effect on noncancer cells [72]. In in vivo experiments on mice, it was found that glycyrrhizin inhibited cancer growth, resulted in weight gain of immune organs and activation of peripheral blood T lymphocytes, and some cytokines, such as IL-2, IL-6, and IL-7 were increased [73]. Besides, it has been reported that licorice extract reverses cisplatin resistance in breast cancer cells by inhibiting cytochrome P450 1B1 enzyme [74].

Licorice is commonly used for cancer therapy of the digestive system, breast cancer, lung cancer, and so on. And it has the advantages of low price and wide distribution.

2.10. Radix Salvia miltiorrhiza (SM, Dan Shen in Chinese). $\mathrm{SM}$ is the dry root and rhizome of Salvia miltiorrhiza in Lamiaceae (Figure 10). The main active ingredients of SM include tanshinone, etc., which are fat-soluble phenanthraquinone compounds with good anticancer activity [75]. SM injection is the most commonly used form in traditional Chinese medicine. It is often used in combination with astragalus.

Studies have shown that ethanol-extracted tanshinone significantly inhibits the activity of gastric adenocarcinoma, prostate cancer, breast cancer, colorectal cancer, and lung adenocarcinoma, respectively [76]. In the study of breast cancer cell line MCF-7, it was found that SM extract reduced the ability of metastasis and invasion of cancer cells by inhibiting the expression of MMP-9 mediated by MAPK/ap1 signal transduction pathway [77]. The dihydrotanshinone in SM can inhibit the proliferation of glioma and promote its apoptosis, possibly through the increased cytochrome $\mathrm{c}$ cytoplasmic level and the activation of caspase 3/9, thereby PARP is lysed [78]. In studies on leukemia cells, it was found that 15,16-dihydrotanshinone I increased the phosphorylation of JNK and the expression of Fas L, which may be the reason for the upregulation of Bad and Bax expressions as well as the activation of caspase $3 / 8 / 9$, leading to the apoptosis of leukemia cells [79]. Salvia acetonitrile extract induces the production of ROS in prostate cancer cells, reduces the expression of cyclin, and increases the expression of apoptosis-related proteins [80]. Besides SM extract, the mixture of SM and astragalus can reduce the expression of 


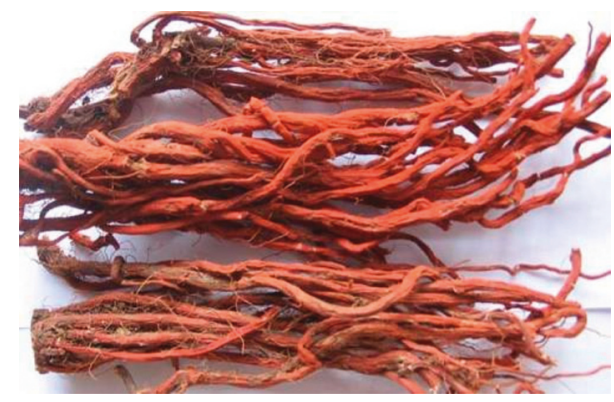

FIgURE 10: Photo of dry root Salvia miltiorrhiza.

TGF-protein and nuclear input protein imp7/8, and the expression of TGF-specific serine/threonine kinase receptor $\mathrm{T} \beta \mathrm{RI}$ and T$\beta \mathrm{RII}$ is also inhibited [81].

$\mathrm{SM}$ and tanshinone have a wide range of anticancer effects to inhibit lung cancer, breast cancer, and cancer of the digestive system. It can also remove blood stasis in cancer patients and is also an excellent choice of anticancer herbs.

\section{Discussion}

Traditional Chinese medicine (TCM) is becoming more and more popular in cancer treatment as a treatment modality or as complementary and alternative medicine (CAM). Conventional chemotherapy and radiation therapy not only kill cancer cells but also cause many side effects to patients. There were also associations with chronic pain, fatigue, and depression. In China, at least a high percentage of cancer patients opt for traditional Chinese medicine to reduce side effects. The Chinese herbal medicine administration method has been continuously improved for thousands of years, and now, it is mainly based on powder, decoction, and injection.

Besides, topical medication is also a good choice, which maintains anticancer drugs in high concentration and longlerm effect on cancer tissue. Our research found that Chinese herbal medicine can change the gene expression of cancer cells. Similar results have been achieved in animals. However, it is not enough to confirm the therapeutic effect of Chinese herbs on cancer. Further trials or clinical trials are needed to prove its efficacy. We speculate that these mechanisms may be related to the role of Chinese herbal medicine in the human body and hope to provide a theoretical basis for future clinical trials. In addition to the ten herbs mentioned above, other Chinese herbs and their preparations, such as Radix isatidis, Pinellia ternata, and Taxus chinensis, have also been recognized by the world in the treatment of cancer. These herbs are widely distributed and more readily available to cancer patients. And the price is much lower than common anticancer drugs, which is affordable to patients.

The usage method is important for the application of Chinese herbal medicine. The correct usage of Chinese herbs can double the effect of cancer therapy with half the effort, while excessive use may have adverse effects on the human body. It has been reported that OD causes acute kidney damage in the treatment of cervical cancer [82]. The reason is the renal toxicity caused by long-term use of OD in the common dose, which improved after a period of discontinuation. Some ingredients in traditional Chinese medicine can also cause drug-induced liver damage [83]. The hepatotoxicity of most traditional Chinese medicines is positively correlated with dose. Different processing methods also affect the level of drug toxicity. Reasonable compatibility and the scientific method should be adopted to reduce its toxicity as far as possible [84]. The decoction used in traditional Chinese medicine is diluted, and the toxicity of the medicine is reduced. Both traditional Chinese medicine tablets and injections have undergone long-term toxicity tests, and patients should follow the doctor's advice or instructions when using them. In short, the vast majority of Chinese herbal medicine, if used correctly, does not produce significant side effects.

\section{Conclusions}

This article introduces ten kinds of Chinese herbal medicines which can be used against cancers in different ways mainly by promoting the apoptosis of cancer cells, inhibiting the activity of cancer cells, and enhancing immunity. In the future, it may be possible to use this natural product with low side effects to fight cancer.

\section{Conflicts of Interest}

The authors declare that there are no conflicts of interest regarding the publication of this paper.

\section{Acknowledgments}

This research was funded by the Major Program of Shandong Province Natural Science Foundation (grant numbers ZR2018ZC1054, ZR2018MH001, and ZR2016HB70). The APC was funded by ZR2018ZC1054 and ZR2018MH001.

\section{References}

[1] F. Bray, J. Ferlay, I. Soerjomataram, R. L. Siegel, L. A. Torre, and A. Jemal, "Global cancer statistics 2018: GLOBOCAN estimates of incidence and mortality worldwide for 36 cancers in 185 countries," CA: A Cancer Journal for Clinicians, vol. 68, no. 6, pp. 394-424, 2018.

[2] J. Qi, A. Zulfiker, C. Li, D. Good, and M. Wei, "The development of toad toxins as potential therapeutic agents," Toxins, vol. 10, no. 8, p. 336, 2018.

[3] F. Qi, L. Zhao, A. Zhou et al., "The advantages of using traditional Chinese medicine as an adjunctive therapy in the whole course of cancer treatment instead of only terminal stage of cancer," Bioscience Trends, vol. 9, no. 1, pp. 16-34, 2015.

[4] L. Jiao, L. Bi, Y. Lu et al., "Cancer chemoprevention and therapy using Chinese herbal medicine," Biological Procedures Online, vol. 20, no. 1, 2018.

[5] H. M. Guo, D. Zhao, and L. Cao, "Hedyotis diffusa Willd promotes the apoptosis of lung cancer cells by inhibiting MAPK pathway," Pharmaceutical and Clinical Research, vol. 27, pp. 5-9, 2019.

[6] A. H. M. Zulfiker, S. M. Hashimi, D. A. Good, I. D. Grice, and M. Q. Wei, "Cane toad skin extract-induced upregulation and increased interaction of serotonin $2 A$ and $D_{2}$ receptors via $G_{q / 11}$ 
signaling pathway in CLU213 cells," Journal of Cellular Biochemistry, vol. 118, no. 5, pp. 979-993, 2017.

[7] A. H. M. Zulfiker, M. Sohrabi, J. Qi, B. Matthews, M. Q. Wei, and I. D. Grice, "Multi-constituent identification in Australian cane toad skin extracts using high-performance liquid chromatography high-resolution tandem mass spectrometry," Journal of Pharmaceutical and Biomedical Analysis, vol. 129, pp. 260-272, 2016.

[8] Y. C. Zeng, S. Y. Liang, and J. H. Wu, "Chemical constituents from hedyotis diffusa and their antitumor activities," Chinese Traditional Patent Medicine, vol. 40, Article ID 17681772, 2018.

[9] H. W. Che, H. N. Yang, and X. C. Tan, "Research progress on the tumor cell inhibition activities of different extraction of Oldenlandia diffusa," Asia-Pacific Traditional Medicine, vol. 15, pp. 191-193, 2019.

[10] Y.-Y. Sunwoo, J.-H. Lee, H. Y. Jung et al., "Oldenlandia diffusa promotes antiproliferative and apoptotic effects in a rat hepatocellular carcinoma with liver cirrhosis," Evidence-Based Complementary and Alternative Medicine, vol. 2015, Article ID 501508, 11 pages, 2015.

[11] T.-W. Chung, H. Choi, J.-M. Lee et al., "Oldenlandia diffusa suppresses metastatic potential through inhibiting matrix metalloproteinase- 9 and intercellular adhesion molecule-1 expression via $\mathrm{p} 38$ and ERK1/2 MAPK pathways and induces apoptosis in human breast cancer MCF-7 cells," Journal of Ethnopharmacology, vol. 195, pp. 309-317, 2017.

[12] J. Zhou and P. Zhao, "Effect of hedyotis diffusa on expressions of apoptosis related protein Fas, caspase 3 and caspase7 in Renca renal cell carcinoma of model mice," Chinese Journal of Biochemical Pharmaceutics, vol. 36, pp. 37-45, 2016.

[13] X. H. Nan, F. Yu, and H. Tian, "Herba hedyotis diffusae induces human bladder cancer T24 cells apoptosis through inhibiting JAK2/STAT3 signaling pathways," Journal of China Prescription Drug, vol. 16, pp. 31-32, 2018.

[14] M. Li, H. Y. Zhang, and H. O. Shao, "Effects of total flavonoids of Oldenlandia diffusa on immune and antioxidant capacity of cervical cancer U14 tumor-bearing mice," Journal of Chinese Medicinal Materials, vol. 6, pp. 1417-1420, 2019.

[15] Y. L. Zhao, "Application of hedyotis diffusa in tumor treatment," Gansu Science and Technology, vol. 34, pp. 141-142, 2018.

[16] H. Y. Zhang, Experimental Study on the Anti-Cervical Cancer Effect of Curcumin and Its Mechanism, Doctor, Central South University, Changsha, China, 2014.

[17] H. F. Liu, Molecular Mechanism of Curcumin Inhibiting TGFInduced EMT in Hepatocellular Carcinoma, Master, University of South China, Hengyang, China, 2017.

[18] X. Wang, J. Deng, J. Yuan et al., "Curcumin exerts its tumor suppressive function via inhibition of NEDD4 oncoprotein in glioma cancer cells," International Journal of Oncology, vol. 51, no. 2, pp. 467-477, 2017.

[19] J. Su, X. Zhou, X. Yin et al., "The effects of curcumin on proliferation, apoptosis, invasion, and NEDD4 expression in pancreatic cancer," Biochemical Pharmacology, vol. 140, pp. 28-40, 2017.

[20] M. D. Yan and X. Y. Cen, "Effects of curcumin on the proliferation and apoptosis of thyroid cancer cell line SW579," Chinese General Medicine, vol. 13, pp. 396-398, 2015.

[21] R. X. Feng, R. G. Cai, and D. D. Xie, "Effects of curcumin on proliferation and apoptosis of oral squamous cell carcinoma Tca8113 cells," Journal of Chengdu Medical College, vol. 14, pp. 25-30, 2019.
[22] L. L. Wei, J. Wang, and S. Tan, "Effects of curcumin on the tumor marker molecular levels and the ability of cervical cancer cell producing nitric oxide of cervical subcutaneous transplantation in mice tumor model," The Chinese Journal of Clinical Pharmacology, vol. 6, pp. 547-549, 2019.

[23] H. H. Wang, D. C. Li, and L. Sun, "Study on the pharmacodynamic components and pharmacological effects of astragalus," Chinese Prescription Drugs, vol. 16, pp. 22-23, 2018.

[24] X. L. Qu, Y. Zeng, and L. Yao, “Astragaloside iv down-regulates the expression of PD-1 and PD-L1 on the inhibition of hela cell invasion and migration in cervical cancer," Journal of Immunology, vol. 34, pp. 850-855, 2018.

[25] W. Li, Y. Kuang, and L. F. Meng, "Mechanism of astragaloside iv on the proliferation, migration and invasion of hela cells in cervical cancer," Pharmacology and Clinic of Traditional Chinese Medicine, vol. 34, pp. 39-42+192, 2018.

[26] J. Zhang, L. Liu, J. Wang, B. Ren, L. Zhang, and W. Li, "Formononetin, an isoflavone from astragalus membranaceus inhibits proliferation and metastasis of ovarian cancer cells," Journal of Ethnopharmacology, vol. 221, pp. 91-99, 2018.

[27] R. Zhou, H. Chen, J. Chen, X. Chen, Y. Wen, and L. Xu, "Extract from astragalus membranaceus inhibit breast cancer cells proliferation via PI3K/AKT/mTOR signaling pathway," BMC Complementary and Alternative Medicine, vol. 18, no. 1, p. 83, 2018.

[28] H. J. Park and S. H. Park, "Induction of apoptosis by ethyl acetate fraction of astragalus membranaceus in human nonsmall cell lung cancer cells: apoptosis induction by astragalus membranaceus," Journal of Pharmacopuncture, vol. 21, no. 4, pp. 268-276, 2018.

[29] L. F. Duan and X. Q. Zhang, "Research progress of astragalus polysaccharide as an immunotherapy for leukemia," Chinese Medicine Guide, vol. 23, pp. 74-76, 2017.

[30] L. X. Wang, W. B. Wu, and Z. H. Xu, "Astragaloside a plays an anti-tumor role by inducing polarization of M1-type macrophages," Chinese Journal of Experimental Formulae, vol. 10, pp. 1-7, 2019.

[31] Q. L. Zhai, X. D. Hu, and J. Xiao, "Mechanism of astragalus polysaccharides in cooperation with cisplatin in inducing apoptosis of cervical cancer siha cells," Guangdong Medical, vol. 39, pp. 1327-1331, 2018.

[32] X. N. Yu, X. G. Feng, and J. M. Zhang, "New progress in studies on chemical constituents and pharmacological effects of ginseng," Ginseng Research, vol. 1, pp. 47-51, 2019.

[33] M. Wang, "Ginsenoside Rh2 promotes apoptosis of human endometrial cancer cells and its mechanism," Southwest National Defense Medicine, vol. 28, pp. 720-723, 2018.

[34] W. Li, W. Liu, and J. J. Zou, "Effect of ginsenoside Rg5 on invasion and migration of gastric cancer BGC-823 cells through miR-125b/STARD13/NEU1 signaling pathway," Chinese Journal of Experimental Formulae, vol. 24, pp. 138142, 2018.

[35] W. Li, W. Liu, and J. J. Zou, "Effects of ginsenoside Rg5 on proliferation, apoptosis and apoptosis-related factors in gastric cancer BGC-823 cells," Chinese Emergency Department of Traditional Chinese Medicine, vol. 27, pp. 955-958, 2018.

[36] R. B. Zhou, S. R. Zhou, and Y. J. Xiang, "Ginsenoside Rg3 induces apoptosis of human lung cancer cell A549 by regulating ROS pathway," The Chinese Medicine, vol. 21, pp. 1145-1150, 2018.

[37] S. G. Sun, J. L. Du, and X. Q. Sun, "Ginsenoside CK inhibits McF-7 cell migration and invasion in breast cancer," 
Lishizhen Medicine and Materia Medica Research, vol. 29, pp. 1068-1070, 2018.

[38] Y. Chen, L. Bi, H. Luo et al., "Water extract of ginseng and astragalus regulates macrophage polarization and synergistically enhances DDP's anticancer effect," Journal of Ethnopharmacology, vol. 232, pp. 11-20, 2019.

[39] Y. Qian, R. R. Huang, and R. Sun, "The immunomodulatory effect of ginsenoside Rh2 on immunocompromised mice," The Medicine Herald, vol. 37, pp. 1446-1454, 2018.

[40] R. T. Zhang, T. Zhou, and X. X. Song, "Research progress on active ingredients and pharmacological action of ganoderma lucidum," Anhui Agricultural Science, vol. 46, p. 1819, 2018.

[41] S. Zolj, M. P. Smith, J. C. Goines et al., "Antiproliferative effects of a triterpene-enriched extract from lingzhi or reishi medicinal mushroom, ganoderma lucidum (agaricomycetes), on human lung cancer cells," International Journal of Medicinal Mushrooms, vol. 20, no. 12, pp. 1173-1183, 2018.

[42] L. Qu, S. Li, Y. Zhuo, J. Chen, X. Qin, and G. Guo, “Anticancer effect of triterpenes from Ganoderma lucidum in human prostate cancer cells," Oncology Letters, vol. 14, pp. 74677472, 2017.

[43] T. J. Rios-Fuller, G. Ortiz-Soto, M. Lacourt-Ventura et al., "Ganoderma lucidum extract (GLE) impairs breast cancer stem cells by targeting the STAT3 pathway," Oncotarget, vol. 9, no. 89, pp. 35907-35921, 2018.

[44] M. M. Martínez-Montemayor, T. Ling, I. J. Suárez-Arroyo et al., "Identification of biologically active ganoderma lucidum compounds and synthesis of improved derivatives that confer anti-cancer activities in vitro," Frontiers in Pharmacology, vol. 10, p. 115, 2019.

[45] J. Su, D. Li, Q. Chen et al., “Anti-breast cancer enhancement of a polysaccharide from spore of ganoderma lucidum with paclitaxel: suppression on tumor metabolism with gut microbiota reshaping," Frontiers in Microbiology, vol. 9, p. 3099, 2018.

[46] S. Zhang, G. Pang, C. Chen et al., "Effective cancer immunotherapy by Ganoderma lucidum polysaccharide-gold nanocomposites through dendritic cell activation and memory T cell response," Carbohydrate Polymers, vol. 205, pp. 192-202, 2019.

[47] X.-P. Chen, W. Li, X.-F. Xiao, L.-L. Zhang, and C.-X. Liu, "Phytochemical and pharmacological studies on Radix Angelica sinensis," Chinese Journal of Natural Medicines, vol. 11, no. 6, pp. 577-587, 2013.

[48] S.-C. Chiu, T.-L. Chiu, S.-Y. Huang et al., "Potential therapeutic effects of N-butylidenephthalide from Radix Angelica Sinensis (Danggui) in human bladder cancer cells," BMC Complementary and Alternative Medicine, vol. 17, no. 1, p. 523, 2017.

[49] M.-C. Chen, W.-L. Hsu, W.-L. Chang, and T.-C. Chou, "Antiangiogenic activity of phthalides-enriched Angelica sinensis extract by suppressing WSB-1/pVHL/HIF- $1 \alpha /$ VEGF signaling in bladder cancer," Scientific Reports, vol. 7, no. 1, p. $5376,2017$.

[50] W.-J. Zhou, S. Wang, Z. Hu, Z.-Y. Zhou, and C.-J. Song, "Angelica sinensis polysaccharides promotes apoptosis in human breast cancer cells via CREB-regulated caspase-3 activation," Biochemical and Biophysical Research Communications, vol. 467, no. 3, pp. 562-569, 2015.

[51] M. Gao, J. H. Zhang, F. X. Zhou et al., "Angelica sinensis suppresses human lung adenocarcinoma A549 cell metastasis by regulating MMPs/TIMPs and TGF- $\beta 1$," Oncology Reports, vol. 27, pp. 585-593, 2012.
[52] W.-F. Zhang, Y. Yang, X. Li et al., "Angelica polysaccharides inhibit the growth and promote the apoptosis of U251 glioma cells in vitro and in vivo," Phytomedicine, vol. 33, pp. 21-27, 2017.

[53] Y.-J. Qiao, J.-H. Shang, D. Wang, H.-T. Zhu, C.-R. Yang, and Y.-J. Zhang, "Research of Panax spp. in kunming institute of botany, CAS," Natural Products and Bioprospecting, vol. 8, no. 4, pp. 245-263, 2018.

[54] T. Wang, R. Guo, G. Zhou et al., "Traditional uses, botany, phytochemistry, pharmacology and toxicology of Panax notoginseng (Burk.) F.H. Chen: a review," Journal of Ethnopharmacology, vol. 188, pp. 234-258, 2016.

[55] S.-L. Hsieh, S. Hsieh, Y.-H. Kuo, J.-J. Wang, J.-C. Wang, and C.-C. Wu, "Effects of Panax notoginseng on the metastasis of human colorectal cancer cells," The American Journal of Chinese Medicine, vol. 44, no. 4, pp. 851-870, 2016.

[56] P. Wang, J. Cui, X. Du et al., "Panax notoginseng saponins (PNS) inhibits breast cancer metastasis," Journal of Ethnopharmacology, vol. 154, no. 3, pp. 663-671, 2014.

[57] Q. Yang, P. Wang, J. Cui, W. Wang, Y. Chen, and T. Zhang, "Panax notoginseng saponins attenuate lung cancer growth in part through modulating the level of Met/miR-222 axis," Journal of Ethnopharmacology, vol. 193, pp. 255-265, 2016.

[58] M.-L. Yu, C.-L. Zhang, D.-D. Yuan, X.-H. Tong, and L. Tao, "Panax notoginseng saponins enhances the cytotoxicity of cisplatin via increasing gap junction intercellular communication," Biological and Pharmaceutical Bulletin, vol. 35, no. 8, pp. 1230-1237, 2012.

[59] B. Kim, E.-Y. Kim, E.-J. Lee et al., "Panax notoginseng inhibits tumor growth through activating macrophage to M1 polarization," The American Journal of Chinese Medicine, vol. 46, no. 6, pp. 1369-1385, 2018.

[60] Y. H. Wang, X. Y. Xu, and S. Q. Yang, "Research progress on pharmacological action and chemical component extraction of Sculellaria barbata," Journal of Mudanjiang Medical University, vol. 38, pp. 116-118, 2017.

[61] L. Zhang, B. Ren, J. Zhang et al., "Anti-tumor effect of Scutellaria barbata D. Don extracts on ovarian cancer and its phytochemicals characterisation," Journal of Ethnopharmacology, vol. 206, pp. 184-192, 2017.

[62] A.-L. Shiau, Y.-T. Shen, J.-L. Hsieh, C.-L. Wu, and C.-H. Lee, "Scutellaria barbata inhibits angiogenesis through downregulation of HIF- $1 \alpha$ in lung tumor," Environmental Toxicology, vol. 29, no. 4, pp. 363-370, 2014.

[63] P. Sun, D. Sun, and X. Wang, "Effects of Scutellaria barbata polysaccharide on the proliferation, apoptosis and EMT of human colon cancer HT29 Cells," Carbohydrate Polymers, vol. 167, pp. 90-96, 2017.

[64] L.-T. Pan, Y. Sheung, W.-P. Guo, Z.-B. Rong, and Z.-M. Cai, "Hedyotis diffusa plus Scutellaria barbata induce bladder cancer cell apoptosis by inhibiting Akt signaling pathway through downregulating miR-155 Expression," EvidenceBased Complementary and Alternative Medicine, vol. 2016, Article ID 9174903, 10 pages, 2016.

[65] L.-H. Wei, J.-M. Lin, J.-F. Chu, H.-W. Chen, Q.-Y. Li, and J. Peng, "Scutellaria barbata D. Don inhibits colorectal cancer growth via suppression of Wnt/ $\beta$-catenin signaling pathway," Chinese Journal of Integrative Medicine, vol. 23, no. 11, pp. 858-863, 2017.

[66] Q. Jiang, Q. Li, H. Chen et al., "Scutellaria barbata D. Don inhibits growth and induces apoptosis by suppressing IL-6inducible STAT3 pathway activation in human colorectal cancer cells," Experimental and Therapeutic Medicine, vol. 10, no. 4, pp. 1602-1608, 2015. 
[67] H. Ye, M. Guo, and Q. Wu, "Effect of polysaccharide from panax terebinata on the activity of caspase-3,8,9 in C26 tumor-bearing mice," Chinese Journal of Gerontology, vol. 23, pp. 5152-5153, 2012.

[68] X. Jiang, S. F. Sun, and Y. Wang, "Research progress on pharmacological action of liquorice," Chemical Industry Times, vol. 31, pp. 25-28, 2017.

[69] S.-C. Lin, P.-Y. Chu, W.-T. Liao et al., "Glycyrrhizic acid induces human MDA-MB-231 breast cancer cell death and autophagy via the ROS-mitochondrial pathway," Oncology Reports, vol. 39, pp. 703-710, 2018.

[70] H. Shen, G. Zeng, B. Sun et al., "A polysaccharide from Glycyrrhiza inflata licorice inhibits proliferation of human oral cancer cells by inducing apoptosis via mitochondrial pathway," Tumour Biology: The Journal of the International Society for Oncodevelopmental Biology and Medicine, vol. 36, no. 6, pp. 4825-4831, 2015.

[71] H. Cai, X. Chen, J. Zhang, and J. Wang, " $18 \beta$-glycyrrhetinic acid inhibits migration and invasion of human gastric cancer cells via the ROS/PKC- $\alpha$ /ERK pathway," Journal of Natural Medicines, vol. 72, no. 1, pp. 252-259, 2018.

[72] S. Khazraei-Moradian, M. Ganjalikhani-Hakemi, A. Andalib, R. Yazdani, J. Arasteh, and G. A. Kardar, "The effect of licorice protein fractions on proliferation and apoptosis of gastrointestinal cancer cell lines," Nutrition and Cancer, vol. 69, no. 2, pp. 330-339, 2017.

[73] P. A. Ayeka, Y. Bian, P. M. Githaiga, and Y. Zhao, "The immunomodulatory activities of licorice polysaccharides (Glycyrrhiza uralensis Fisch.) in CT 26 tumor-bearing mice," BMC Complementary and Alternative Medicine, vol. 17, no. 1, p. 536, 2017.

[74] R. Sharma, L. Gatchie, I. S. Williams et al., "Glycyrrhiza glabra extract and quercetin reverses cisplatin resistance in triplenegative MDA-MB-468 breast cancer cells via inhibition of cytochrome P450 1B1 enzyme," Bioorganic \& Medicinal Chemistry Letters, vol. 27, no. 24, pp. 5400-5403, 2017.

[75] L. Liu, G. J. Zhang, and Z. H. Lou, "Research progress of active components of Salvia miltiorrhiza in preventing and treating malignant tumor," Chinese Journal of Traditional Chinese Medicine, vol. 33, pp. 2472-2475, 2018.

[76] B. Sung, H. S. Chung, M. Kim et al., "Cytotoxic effects of solvent-extracted active components of Salvia miltiorrhiza Bunge on human cancer cell lines," Experimental and Therapeutic Medicine, vol. 9, no. 4, pp. 1421-1428, 2015.

[77] J.-M. Kim, E.-M. Noh, H.-K. Song et al., "Salvia miltiorrhiza extract inhibits TPA-induced MMP-9 expression and invasion through the MAPK/AP-1 signaling pathway in human breast cancer MCF-7 cells," Oncology Letters, vol. 14, no. 3, pp. 3594-3600, 2017.

[78] Y. Cao, B. Huang, and C. Gao, "Salvia miltiorrhiza extract dihydrotanshinone induces apoptosis and inhibits proliferation of glioma cells," Bosnian Journal of Basic Medical Sciences, vol. 17, pp. 235-240, 2017.

[79] J.-J. Liu, H.-H. Wu, T.-H. Chen, W. Leung, and Y.-C. Liang, "15,16-dihydrotanshinone I from the functional food Salvia miltiorrhiza exhibits anticancer activity in human HL-60 leukemia cells: in vitro and in vivo studies," International Journal of Molecular Sciences, vol. 16, no. 8, pp. 19387-19400, 2015.

[80] J. Lee, B. Y. Choi, and Y.-S. Keum, "Acetonitrile extract of Salvia miltiorrhiza radix exhibits growth-inhibitory effects on prostate cancer cells through the induction of cell cycle arrest and apoptosis," Oncology Letters, vol. 13, no. 5, pp. 2921-2928, 2017.
[81] C. Wu, H. Kan, M. Hu et al., "Compound astragalus and Salvia miltiorrhiza extract inhibits hepatocarcinogenesis via modulating TGF- $\beta / \mathrm{T} \beta \mathrm{R}$ and Imp7/8," Experimental and Therapeutic Medicine, vol. 16, pp. 1052-1060, 2018.

[82] M. Y. Hu, D. H. Huang, and Y. Fu, "One case of acute kidney injury caused by herba ophiopogonis," Herald of Medicine, vol. 37, pp. 1424-1425, 2018.

[83] Z. D. Shi, "The pathogenesis, clinical characteristics and prevention and treatment strategies of liver injury related to Chinese herbal medicine," Chinese Journal of Modern Medicine, vol. 19, pp. 101-104, 2017.

[84] C. Li, S. M. Hashimi, A. Good, S. Cao, W. Duan, and M. Q. Wei, "Chansu inhibits the expression of cortactin in colon cancer cell lines in vitro and in vivo," BMC Complementary and Alternative Medicine, vol. 15, no. 1, p. 207, 2015. 


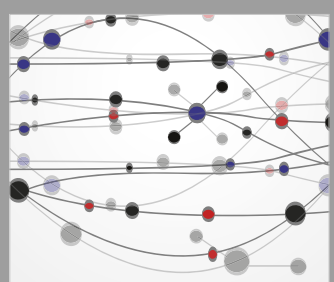

The Scientific World Journal
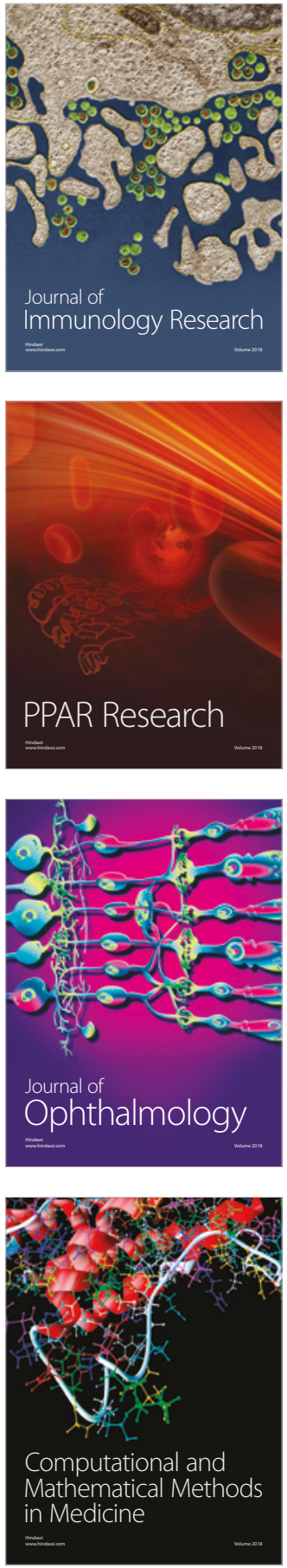

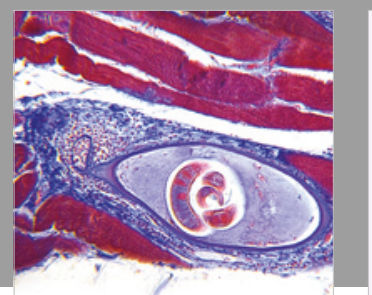

Gastroenterology Research and Practice

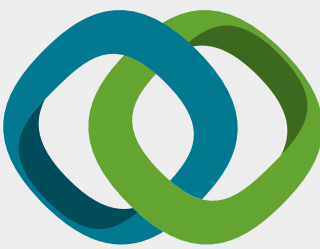

\section{Hindawi}

Submit your manuscripts at

www.hindawi.com
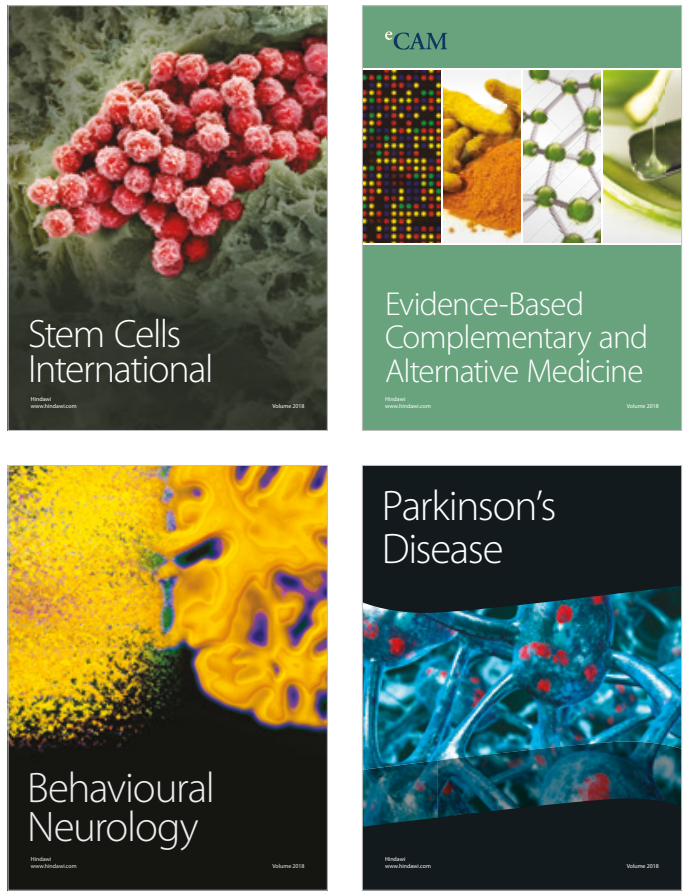

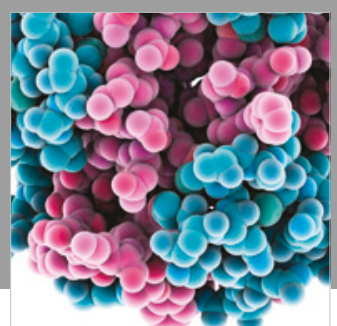

ournal of

Diabetes Research

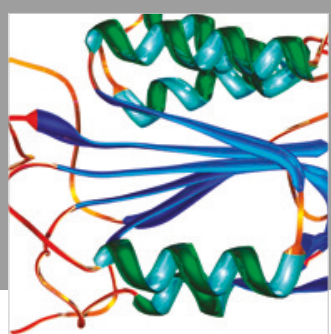

Disease Markers
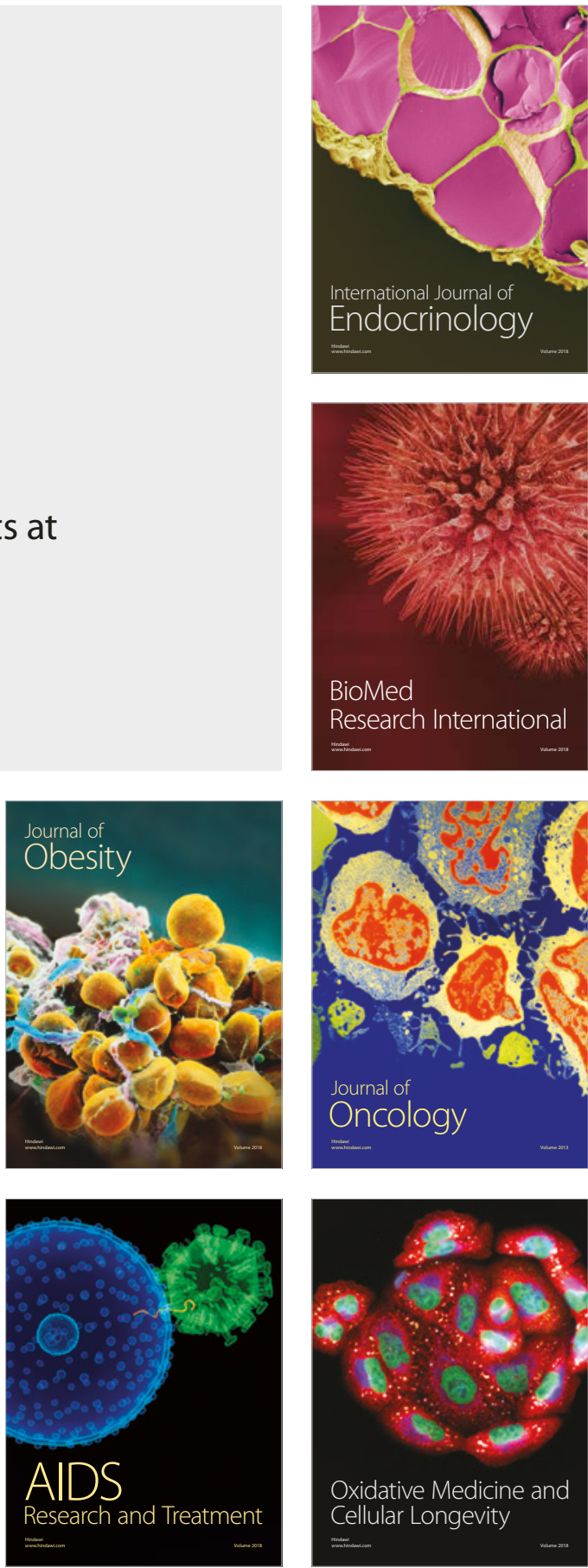